\title{
Surface modification of Co-doped ZnO nanocrystals and its effects on the magnetic properties
}

\author{
A. S. Pereira \\ Departamento de Química and CICECO, Universidade de Aveiro, P-3810-193 Aveiro, Portugal
}

A. O. Ankiewicz ${ }^{\text {a) }}$

I3 N-Institute for Nanostructures, Nanomodelling and Nanomanufacturing and Departamento de Física, Universidade de Aveiro, P-3810-193 Aveiro, Portugal and Institut für Experimentelle Physik II, Universität Leipzig, D-04103 Leipzig, Germany

W. Gehlhoff and A. Hoffmann

Institut für Festkörperphysik, Technische Universität Berlin, D-10623 Berlin, Germany

S. Pereira and T. Trindade

CICECO, Universidade de Aveiro, P-3810-193 Aveiro, Portugal

\section{Grundmann}

Institut für Experimentelle Physik II, Universität Leipzig, D-04103 Leipzig, Germany

\section{C. Carmo and N. A. Sobolev}

I3 N-Institute for Nanostructures, Nanomodelling and Nanomanufacturing and Departamento de Física, Universidade de Aveiro, P-3810-193 Aveiro, Portugal

(Presented on 6 November 2007; received 11 September 2007; accepted 25 October 2007; published online 18 March 2008)

\begin{abstract}
A series of chemically prepared $\mathrm{Co}^{2+}$-doped $\mathrm{ZnO}$ colloids has been surface modified either by growing shells of $\mathrm{ZnSe}$ or by the in situ encapsulation in poly(styrene). The surface modification effects using these two distinct chemical strategies on the magnetic properties of the nanocrystals were probed by electron paramagnetic resonance (EPR). Structural characterization by means of $\mathrm{x}$-ray diffraction and transmission electron microscopy gave no evidence of second phase formation within the detection limits of the used equipment. The EPR analysis was carried out by simulations of the powderlike EPR spectra. The results confirm that in the core of these nanocrystals Co was incorporated as $\mathrm{Co}^{2+}$, occupying the $\mathrm{Zn}^{2+}$ sites in the wurtzite structure of $\mathrm{ZnO}$. Additionally we identify two Co signals stemming from the nanocrystals' shell. The performed surface modifications clearly change the relative intensity of the EPR spectrum components, revealing the core and shell signals. (C) 2008 American Institute of Physics. [DOI: 10.1063/1.2833300]
\end{abstract}

\section{INTRODUCTION}

Magnetic semiconductors combining properties and functionalities of both semiconductors and ferromagnets are driving an enormous scientific activity due to their potential applications in spintronics. ${ }^{1,2} \mathrm{ZnO}$ based diluted magnetic semiconductors are particularly promising due to their large band gap and to the possibility of achieving room temperature charge mediated ferromagnetism. Some groups have reported ferromagnetism in transition metal doped $\mathrm{ZnO}$ systems with Curie temperatures $T_{C}$ ranging from 30 to $550 \mathrm{~K},{ }^{3-13}$ while others have found antiferromagnetic, spin glass, or paramagnetic behavior. ${ }^{14-19}$ Thus, the topic is still controversial and further investigations are needed to clarify these materials' magnetic properties that are very sensitive to the preparation method. Simultaneously, there have been great efforts toward achieving high quality $\mathrm{ZnO}$ nanocrystals $(\mathrm{NCs}),{ }^{6,7}$ where surface related effects are especially relevant due to the high surface-to-volume ratio. In particular, for metal doped NCs, little is known about the effects of chemical surface modification strategies on doping. We

a)Electronic mail: amelia@ua.pt. present a detailed investigation of the influence of the surface modification, namely, polymer encapsulation and inorganic capping, on the magnetic properties of Co-doped $\mathrm{ZnO}$ colloidal NCs. The magnetic and structural properties were probed by electron paramagnetic resonance (EPR), x-ray diffraction (XRD), and transmission electron microscopy (TEM).

\section{SAMPLE PREPARATION}

All chemicals were supplied by Aldrich, except ethyl acetate (Lab-Scan). All the chemicals were used as received, except the organic solvents which were dried over molecular sieves. The doped $\mathrm{ZnO}$ colloids were synthesized by the dropwise addition of $33.3 \mathrm{~cm}^{3}$ of absolute ethanol containing tetramethylammonium hydroxide $\left(\mathrm{N}\left(\mathrm{CH}_{3}\right)_{4} \mathrm{OH} \cdot 5 \mathrm{H}_{2} \mathrm{O}\right.$ : $0.552 \mathrm{~mol} \mathrm{dm}^{-3}$ ) to $\mathrm{Zn}^{2+}: \mathrm{Co}^{2+}$ dimethylsulfoxide (DMSO) solutions. ${ }^{7}$ These solutions were prepared by adding $\mathrm{Co}\left(\mathrm{CH}_{3} \mathrm{COO}\right)_{2} \cdot 6 \mathrm{H}_{2} \mathrm{O}$ to $100 \mathrm{~cm}^{3}$ of a DMSO $0.101 \mathrm{~mol} \mathrm{dm}^{-3}$ solution in $\mathrm{Zn}\left(\mathrm{CH}_{3} \mathrm{COO}\right)_{2} \cdot 2 \mathrm{H}_{2} \mathrm{O}$ to achieve a $5 \%$ nominal mole doping. To extract the Co-doped $\mathrm{ZnO}$ NCs as powders (sample A), ethyl acetate was added to the colloid; the formed precipitate was centrifuged and washed 


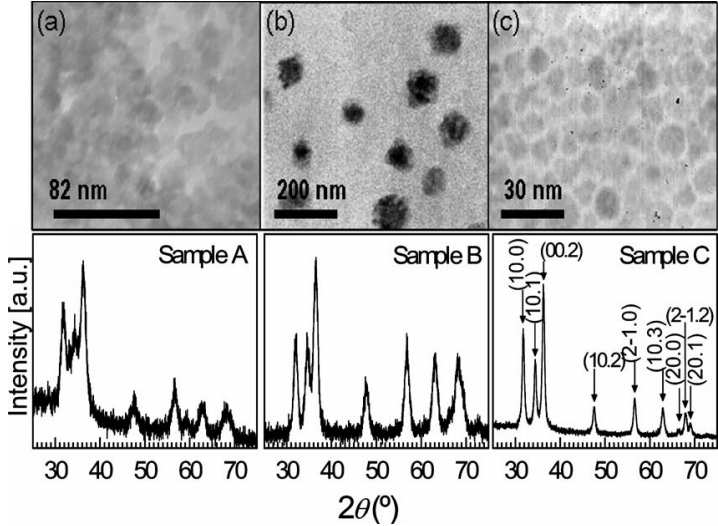

FIG. 1. TEM images of (a) ZnO:Co NCs (sample A), (b) polystyrene/ $\mathrm{ZnO}$ :Co nanocomposites (sample B), and (c) ZnO:Co NCs capped with $\mathrm{ZnSe}$ (sample C). The inferior part of the figure shows the XRD results for all three samples. The peak identification given for sample $\mathrm{C}$ is the same as for samples A and B.

with ethyl acetate and methanol. Trioctylphosphine oxide (TOPO) capped Co-doped $\mathrm{ZnO}$ NCs, obtained after thermal treatment of part of sample A at $150{ }^{\circ} \mathrm{C}$ for $4 \mathrm{~h}$ under a nitrogen stream, were thoroughly washed with methanol and subjected to chemical surface modification by two distinct routes.

(1) A miniemulsion polymerization technique was employed to prepare poly(styrene) (PS) nanocomposites containing $\mathrm{Co}^{2+}$-doped $\mathrm{ZnO}$ NCs. The miniemulsion was prepared by mixing a solution containing $\mathrm{H}_{2} \mathrm{O}$ $(17.7399 \mathrm{~g}), \mathrm{NaHCO}_{3}(11.9 \mathrm{mg})$, and $\mathrm{SDS}(57.5 \mathrm{mg})$, with another solution containing $\mathrm{ZnO}: \mathrm{Co}(6 \mathrm{mg})$, styrene $(4 \mathrm{ml})$, hexadecane $(82.1 \mathrm{mg})$, and AIBN $(11.5 \mathrm{mg})$. These two solutions were mixed and stirred for $30 \mathrm{~min}$ and sonicated for $5 \mathrm{~min}$. The reacting mixture was held $2 \mathrm{~h}$ at $70{ }^{\circ} \mathrm{C}$ after being purged with $\mathrm{N}_{2}$ for $20 \mathrm{~min}$. The final nanocomposite (sample B) was retrieved through centrifugation and washed with water to remove excess of PS.

(2) In another strategy, the surface modification of the NCs was achieved by dispersing them in $2 \mathrm{~cm}^{3}$ of TOP and heating the mixture up to $280{ }^{\circ} \mathrm{C}$ under a $\mathrm{N}_{2}$ stream. An excess of TOPSe $\left(2 \mathrm{~mol} \mathrm{dm}^{-3}\right)$ was then injected into the hot mixture and the reaction was kept at this temperature for $4 \mathrm{~h}$, under $\mathrm{N}_{2}$. During this process, the formation of $\mathrm{ZnSe}$ at the $\mathrm{ZnO}$ surfaces was monitored by UV/visible spectroscopy. The extraction and washing of the NCs (sample C) were performed with a mixture (1:3) of methanol and isopropanol.

\section{STRUCTURAL CHARACTERIZATION}

In Fig. 1 we show the TEM images together with the XRD measurements for all three samples. XRD evidenced the formation of $\mathrm{ZnO}$ NCs with a hexagonal wurtzite crystal structure for all samples. No Co clusters or Co-related complexes were detected, at least within the sensitivity of the apparatus. Excluding instrument broadening effects [using a certified standard NIST SRM660a ( $\mathrm{LaB}_{6}$ powder)] and ap-

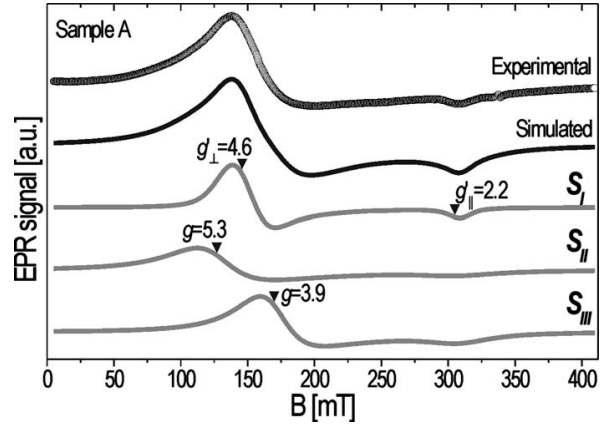

FIG. 2. Experimental EPR spectrum taken at $10 \mathrm{~K}$ of $\mathrm{ZnO}: \mathrm{Co}$ (nominal Co content of $5 \%$ ) nanocrystals along with the respective simulation carried out by adding the EPR signals $S_{\mathrm{I}}, S_{\mathrm{II}}$, and $S_{\mathrm{III}}$.

plying the Scherrer equation, we estimate the crystallite size as a function of the peak width, yielding average diameters of $6 \pm 2,6 \pm 2$, and $12 \pm 3 \mathrm{~nm}$ for the NCs in samples A, B, and $\mathrm{C}$, respectively. UV/visible absorption of the $5 \% \mathrm{Co}-$ doped $\mathrm{ZnO}$ NCs dispersed in dimethylsulfoxide exhibits the characteristic optical spectrum of a colloid containing $\mathrm{ZnO}$ $\mathrm{NCs}$, revealing the well known absorption spectrum of $\mathrm{Co}^{2+}$ ions in tetrahedral coordination geometry. ${ }^{19}$ The TEM image shown in Fig. 1(b) exhibits that each nanocomposite particle of sample B results from the PS encapsulation of a group of $\mathrm{ZnO}$ :Co NCs forming morphologically well-defined particles with a $\mathrm{ZnO}$ phase (dark spots) dispersed in round shaped PS beads, in agreement with our previous findings for other systems. ${ }^{20,21}$ The absorbance spectrum of sample C exhibits the development of an optical feature in the visible region of the spectrum $(430-450 \mathrm{~nm})$, which is ascribed to the formation of ZnSe. Figure 1(c) shows a TEM image obtained after 20 min reaction, evidencing fairly morphologically homogenous nanoparticles.

\section{EPR ANALYSIS}

EPR measurements were performed in the $X$ $(\sim 9.5 \mathrm{GHz})$ and $Q$ (and $\sim 34 \mathrm{GHz})$ bands for all $\mathrm{ZnO} \mathrm{NC}$ samples, at temperatures between 4 and $300 \mathrm{~K}$, using a Bruker ESP 300E spectrometer equipped with Oxford Instruments continuous flow helium cryostats. In the case of substitutional $\mathrm{Co}^{2+}\left(3 d^{7}\right)$ on the $\mathrm{Zn}$ sites the ground state is the orbital singlet ${ }^{4} A_{2}$ and, due to the strong hexagonal crystal field, the zero-field splitting is much larger than the Zeeman energy; consequently only the electron spin transitions within the $\left| \pm \frac{1}{2}\right\rangle$ doublet can be observed in the available magnetic field range. Thus, we can describe the spectra within a good approach by the formalism presented in Ref. 16 considering an effective electronic spin of $S^{\prime}=1 / 2$ and a nuclear spin of $I=7 / 2$. We simulate the EPR powder spectra by diagonalization of the spin Hamiltonian ( $\mathrm{SH}$ ) and fully integrating over the space angles using the EASYSPIN software package. ${ }^{22}$ Illustratively, the simulations of the three EPR signals for sample A are shown in Fig. 2. Figure 3 presents the final result of the simulations of the EPR spectra for all samples with the respective experimental traces. The analysis proves that part of the spectra $\left(S_{I}\right)$ is due to substitutional $\mathrm{Co}^{2+}$ with effective SH parameters $g_{\|}^{\prime}=2.2, g_{\perp}^{\prime}=4.6$, $A_{\|}^{\prime}=16.0 \times 10^{-4} \mathrm{~cm}^{-1}$, and $A_{\perp}^{\prime}=3.0 \times 10^{-4} \mathrm{~cm}^{-1}$, in agree- 


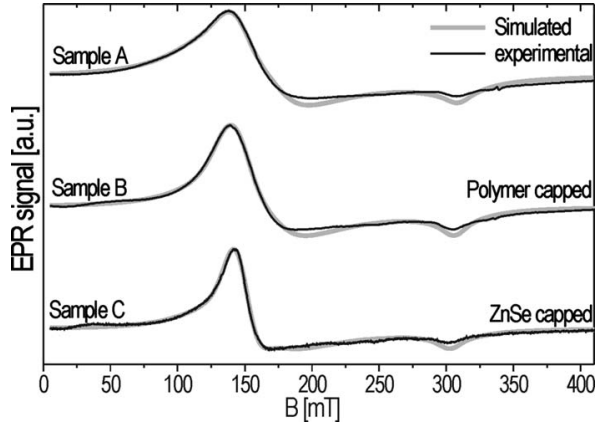

FIG. 3. Experimental EPR spectra taken at $10 \mathrm{~K}$ of $\mathrm{ZnO}$ :Co (nominal $\mathrm{Co}$ content of 5\%) raw nanocrystals (sample A), of that after polymer encapsulation (sample B), or after reaction with TOPSe (sample C). In light gray are plotted the respective simulations carried out by adding the EPR signals $S_{\mathrm{I}}$, $S_{\text {II }}$, and $S_{\text {III }}$

ment with the bulk values, ${ }^{23}$ where $g^{\prime}$ is the effective $g$ value, $A^{\prime}$ is the effective hyperfine constant, and the symbols $\|$ and $\perp$ designate the applied magnetic field parallel and perpendicular to the $c$ axis, respectively. Additionally, there are two other Co lines, $S_{\text {II }}$ and $S_{\text {III }}$, which are caused by a locally distorted environment and can be described by $g_{\|}^{\prime}$ $=2.2$ and $g_{\perp}^{\prime}=5.3$, and $g_{\|}^{\prime}=2.2$ as well as $g_{\perp}^{\prime}=3.9$ for $S_{\mathrm{II}}$ and $S_{\text {III }}$, respectively, as confirmed by $Q$-band measurements (not shown here), which have a higher spectral resolution. The surface exchange reaction with TOPSe occurring during the preparation of sample $\mathrm{C}$, in which $\mathrm{ZnSe}$ is formed at the expense of $\mathrm{Zn}$ on the shell of the NCs, partially removes the $S_{\text {II }}$ and $S_{\text {III }}$ signals, revealing a core-shell structure. As expected, this effect is not so pronounced for sample B, as the surface is not removed by the PS encapsulation.

\section{CONCLUDING REMARKS}

We have incorporated Co ions in $\mathrm{ZnO}$ colloidal NCs and subsequently modified their surfaces by PS encapsulation or by forming a $\mathrm{ZnSe}$ shell on their surface. By EPR spectroscopy we are able to distinguish three different signals: one coming from substitutional $\mathrm{Co}^{2+}$ in the $\mathrm{NC}$ core with $\mathrm{SH}$ parameters similar to the bulk ones and two other Co lines caused by locally distorted environment in the shell. No evidence of ferromagnetism was found in this series of samples. The core-shell structure is also proved by partially removing the shell signal when $\mathrm{ZnSe}$ is formed at the expense of $\mathrm{Zn}$ on the shell of the NCs, i.e., "etching" the shell. As the surface shell is not removed by the PS encapsulation, this effect is not so pronounced for this surface modification procedure.

\section{ACKNOWLEDGMENTS}

This work has been supported by the SANDiE Network of Excellence of the EU, by the Fundação para a Ciência e a Tecnologia of Portugal, and FEDER for funding (Project Nos. PTDC/FIS/72843/2006 and PTDC/QUI/67712/2006; and Grant Nos. SFRH/BD/16985/2004 and SFRH/BD/ 21659/2005).

${ }^{1}$ C. Liu, F. Yun, and H. Morkoç, J. Mater. Sci.: Mater. Electron. 16, 555 (2005).

${ }^{2}$ T. Dietl, J. Phys.: Condens. Matter 19, 165204 (2007).

${ }^{3}$ S. W. Jung, S.-J. An, G.-C. Yi, C. U. Jung, S.-I. Lee, and S. Cho, Appl. Phys. Lett. 80, 4561 (2002).

${ }^{4}$ Y. W. Heo, M. P. Ivill, K. Ip, D. P. Norton, and S. J. Pearton, Appl. Phys. Lett. 84, 2292 (2004).

${ }^{5}$ D. P. Norton, S. J. Pearton, A. F. Herbard, N. Theodoropoulou, L. A. Boatner, and R. G. Wilson, Appl. Phys. Lett. 82, 239 (2003).

${ }^{6}$ N. S. Norberg, K. R. Kittilstved, J. E. Amonette, R. K. Kukkadapu, D. A. Schwartz, and D. R. Gamelin, J. Am. Chem. Soc. 126, 9387 (2004).

${ }^{7}$ D. A. Schwartz, N. S. Norberg, Q. P. Nguyen, J. M. Parker, and D. R. Gamelin, J. Am. Chem. Soc. 125, 13205 (2003).

${ }^{8}$ H.-J. Lee, S.-Y. Jeong, C. R. Cho, and C. H. Park, Appl. Phys. Lett. 81, 4020 (2002).

${ }^{9}$ Y. M. Cho, W. K. Choo, H. Kim, D. Kim, and Y. E. Ihm, Appl. Phys. Lett. 80, 3358 (2002).

${ }^{10}$ P. Sharma, A. Gupta, K. V. Rao, F. J. Owens, R. Sharma, R. Ahuja, J. M. Osorio Guillen, B. Johansson, and G. A. Gehring, Nat. Mater. 2, 673 (2003).

${ }^{11}$ K. Ueda, H. Tabat, and T. Kawai, Appl. Phys. Lett. 79, 988 (2001).

${ }^{12}$ T. Wakano, N. Fujimura, Y. Morinaga, N. Abe, A. Ashida, and T. Ito, Physica E (Amsterdam) 10, 260 (2001).

${ }^{13}$ H. Saeki, H. Tabata, and T. Kawai, Solid State Commun. 120, 439 (2001).

${ }^{14}$ J. H. Kim, H. Kim, Y. E. Ihm, and W. K. Choo, J. Appl. Phys. 92, 6066 (2002).

${ }^{15}$ T. Fukumura, Z. Jin, M. Kawasaki, T. Shono, T. Hasegawa, S. Koshihara, and H. Koinuma, Appl. Phys. Lett. 78, 958 (2001).

${ }^{16}$ A. O. Ankiewicz, W. Gehlhoff, E. M. Kaidashev, A. Rahm, M. Lorenz, M. Grundmann, M. C. Carmo, and N. A. Sobolev, J. Appl. Phys. 101, 024324 (2007).

${ }^{17}$ M. H. Kane, K. Shalini, C. J. Summers, R. Varatharajan, J. Nause, C. R. Vestal, Z. J. Zhang, and I. T. Ferguson, J. Appl. Phys. 97, 023906 (2005).

${ }^{18}$ M. Diaconu, H. Schmidt, A. Pöppl, R. Böttcher, J. Hoentsch, A. Rahm, H. Hochmuth, M. Lorenz, and M. Grundmann, Superlattices Microstruct. 38, 413 (2005).

${ }^{19}$ N. Volbers, H. Zhou, C. Knies, D. Pfisterer, J. Sann, D. M. Hofmann, and B. K. Meyer, Appl. Phys. A: Mater. Sci. Process. 88, 153 (2007).

${ }^{20}$ A. C. C. Esteves, A. Barros-Timmons, T. Monteiro, and T. Trindade, J. Nanosci. Nanotechnol. 5, 766 (2005).

${ }^{21}$ M. A. Martins, M. C. Neves, A. C. C. Esteves, P. I. Girginova, A. J. Guiomar, V. S. Amaral, and T. Trindade, Nanotechnology 18, 215609 (2007).

${ }^{22}$ S. Stoll and A. Schweiger, J. Magn. Reson. 178, 42 (2006).

${ }^{23}$ T. L. Estle and M. De Witt, Bull. Am. Phys. Soc. 6(5), 445 (1961). 